\title{
SpS1-POSTER PAPERS
}

High resolution atomic spectroscopy at Lund Observatory

R. Blackwell-Whitehead, H. Hartman, H. Nilsson

Measurement of the sulphur abundance in halo stars by using Mult. 3 at $1045 \mathrm{~nm}$

E. Caffau, L. Sbordone, H. Ludwig, P. Bonifacio, M. Spite

A far-infrared AKARI-FIS survey of post-AGB stars and (proto) planetary nebluae

N. Cox, P. Garcia-Lario, A. Garcia-Hernandez, A. Manchado

PO molecular line prospects for sunspot umbrae

A.A. de Almeida, G.C. Sanzovo, R. Boczko

$\mathrm{K}$ and L-band spectroscopy of Be stars

A. Granada, M.L. Arias, L.S. Cidale

VLT-CRIRES Observations of Eta Carinae ejecta: Weigelt blobs and Sr filament

H. Hartman, J. Groh, T.R. Gull, H.U. Käufl, F. Kerber, V. Letokhov, K.R. Nielsen

Submillimeter spectral line observations of early stage protostellar candidates

T. Hill, M.R. Cunningham, V. Minier

Modelling of circumstellar dust around symbiotic Miras in the infrared

T. Jurkic, D. Kotnik-Karuza

Standards and reference data for calibration of infrared spectrographs

F. Kerber, M. Aldenius, P. Bristow, G. Nave, Y. Ralchenko, C.J. Sansonetti

Infrared lines in NLTE model atmospheres of hot stars

J. Kubát, B. Šurlan

CRIRES in self-aligning spectrograph mode, a fundamental improvement for quantitative stellar spectroscopy

H.U. Käufl, J.-F. Picard, A. Smette, F. Kerber, U. Seemann

CRIRES-POP: A new high resolution near-IR spectral library

T. Lebzelter, S. Bagnulo, T. Dall, H. Hartman, G. Hussain, U. Käufl, M. Lederer, F. Nieva, N. Przybilla, S. Ramsay, A. Seifahrt, A. Smette, S. Uttenthaler, G.M. Wahlgren

Dust in S stars

S. Lorenz-Martins, H.S. de Almeida

Far-infrared spectroscopic imaging of interstellar material around Eta Carinae

H. Matsuo, T. Arai, T. Nitta, A. Kosaka

Infrared integral field spectrometer observations of molecular hydrogen lines on the planetary nebula NGC 7293

M. Matsuura

Evolution among extreme Carbon stars and post-AGBs

A.F.P. Sánchez, A.B. de Mello, S. Lorenz-Martins

CRIRES: A new era in high-resolution infrared spectroscopy

U. Seemann, H.U. Käufl, A. Reiners

A view on dead $\mathrm{OH} / \mathrm{IR}$ stars

T.C.K. Silva, S. Lorenz-Martins, R. Ortiz, A.F.P. Sánchez

Heavy element abundances of cool supergiants from near-IR spectra

G.M. Wahlgren, K.G. Carpenter, R.P. Norris 